\title{
Aplicação de BIM e SIG para avaliação de impacto ambiental em estudo de traçado rodoviário
}

Projetos de infraestrutura rodoviária demandam extensas análises e estudos a fim de se obter empreendimentos que atendam às necessidades humanas com o mínimo impacto gerado ao meio ambiente em que se localizam. A Avaliação de Impactos Ambientais tradicionalmente é aplicada após a determinação da alternativa de traçado, porém durante o estudo de alternativas locacionais há a necessidade de se considerar os impactos gerados por cada alternativa, de modo a definir o traçado de menor impacto ambiental. Neste sentido, o presente trabalho apresenta uma aplicação de programas de modelagem da informação em conjunto com sistemas de informações georreferenciadas para uso Avaliação de Impactos Ambientais de estudos de alternativas locacionais rodoviárias. Em vista disso, este trabalho visa verificar a aplicabilidade de tecnologias BIM e SIG, por meio do estudo de caso aplicado ao trecho da rodovia BR-135/BA, entre os km 207 a 212 no município de São Desidério na Bahia, onde foram empregados fluxos de trabalho utilizando programas computacionais de ambas as tecnologias, para a geração de alternativas locacionais com avaliação de seu respectivo impacto ambiental. Os resultados obtidos pela aplicação das tecnologias BIM e SIG na geração de alternativas de traçado, permitiram a verificação de que a tecnologia SIG apresenta potencial para uso em avaliação de impactos de maneira conjunta à elaboração do projeto em CAD paramétrico dependendo dos pesos e critérios empregados na otimização, enquanto a tecnologia BIM empregada ainda não possui a capacidade de processamento necessária para a avaliação de todas as restrições ambientais consideradas.

Palavras-chave: Tecnologia BIM; Avaliação de Impacto; Estudo de Traçado Rodoviário; SIG; Alternativas locacionais.

\section{BIM and GIS technologies applied to environmental impact analisys during roadway route planning}

\begin{abstract}
Road infrastructure projects require extensive analysis to meet human needs with a minimal impact on the environment. Environmental Impact Assessment is traditionally applied after the road alternative is determined, however, during the alignment planning there is a need to consider the impacts generated by each alternative, to define the alignment with the least impact. Thus, this paper presents an application of Information Modeling programs in conjunction with Georeferenced Information Systems for Environmental Impact Assessment of road alignment planning studies. Therefore, this thesis aims to verify the applicability of BIM and GIS technologies, through the case study of a segment in BR-135/BA, between km 207 to 212, in the municipality of São Desidério, Bahia. Different workflows in computer programs of both technologies were applied in the generation of alignment alternatives. The results obtained by the application of BIM and GIS technologies in the alignment optimization, were analyzed in in terms of estimated environmental impact. It was found that GIS technology has the potential for use in impact assessment if used in conjunction with project design, depending on the weights and criteria employed in the optimization. Whereas, the tested BIM technology does not yet have the processing capacity required to evaluate all the environmental restrictions considered in this case study.
\end{abstract}

Keywords: BIM Technology; Environmental Impact Assessment; Roadway Alignment Planning; GIS; Road Location Alternatives.

Topic: Tecnologia, Modelagem e Geoprocessamento

Received: 02/04/2021

Approved: 29/04/2021

Reviewed anonymously in the process of blind peer.

Alessandra Costa Gosch (D)

Universidade Federal do Paraná, Brasil

http://lattes.cnpq.br/7401938280106461

http://orcid.org/0000-0001-9273-6629

alecgosch@gmail.com

Eduardo Ratton (ii)

Universidade Federal do Paraná, Brasil

http://lattes.cnpq.br/1582314027487253

http://orcid.org/0000-0002-1309-0063

ratton.eduardo@gmail.com

Sandra Mara Pereira de Queiroz (iD

Universidade Federal do Paraná, Brasil

http://lattes.cnpq.br/3808625947714374

http://orcid.org/0000-0002-9929-6205

sandraqueiroz30@yahoo.com.br
Referencing this:

GOSCH, A. C.; RATTON, E.; QUEIROZ, S. M. P.. Aplicação de BIM e SIG para avaliação de impacto ambiental em estudo de traçado rodoviário. Revista Ibero Americana de Ciências Ambientais, v.12, n.4, p.697-711, 2021. DOI: http://doi.org/10.6008/CBPC21796858.2021.004.0054 


\section{INTRODUÇÃO}

Os projetos de infraestrutura rodoviária são constituídos de estudos em diversas fases, sendo o estudo de alternativas locacionais de traçado uma fase determinante para o impacto ambiental a ser causado pelo empreendimento. Entretanto, usualmente trata-se de uma fase que ocorre previamente à avaliação de impactos ambientais (AIA), visto que a AIA é realizada em função do projeto já definido. Assim, a determinação da alternativa locacional usualmente não considera avaliação estruturada de impactos em sua definição.

São necessárias extensas análises a fim de se obter um projeto rodoviário que melhor supra às necessidades das populações atendidas, gerando o mínimo impacto ao ambiente em que se localizam. Tratam-se de procedimentos de combinação de elementos sociais divergentes em um mesmo espaço, nos quais deve-se garantir o objetivo estratégico do desenvolvimento sustentável ao adequar os meios de exploração dos recursos naturais às limitações do meio ambiente, de modo a impedir ou minimizar os impactos ambientais e cumprir com as diretrizes de conservação que visam a preservação e proteção do meio ambiente (PIMENTA et al., 2014).

Os riscos ambientais envolvidos em obras rodoviárias, para serem adequadamente mensurados requerem obtenção e análise de informações sobre os principais componentes dos meios afetados. Assim, este trabalho se propõe a empregar fluxos de trabalho que permitam um nível de avaliação dos impactos já durante a elaboração da alternativa locacional de traçado rodoviário, de modo a resultar em traçados com menores riscos ambientais aos meios afetados.

Para tanto, procurou-se aferir a validade da aplicação de programas de modelagem da informação em conjunto com sistemas de informações georreferenciadas, em determinado estudo de caso de um segmento rodoviário em implantação da BR - 135 na Bahia, entre os km 207 a 212 no município de São Desidério.

A metodologia aplicada para estudo de alternativa locacional da citada rodovia foi a aplicação de tecnologias de Modelagem de Informações da Construção (BIM) e Sistemas de Informações Geográficas (SIG) em diferentes fluxos de trabalho para otimização de traçados viários visando o menor impacto ambiental.

Destarte, os objetivos deste estudo são aplicar as tecnologias BIM e SIG para a geração de alternativas de traçado com Avaliação de Impacto Ambiental, com o objetivo específico de verificar a aplicabilidade em Estudos de Traçados Rodoviário, por meio do Estudo de Caso aplicado ao de trecho da rodovia BR-135 na Bahia, entre os km 207 a 212 no município de São Desidério na Bahia. Ademais, objetivouse ainda determinar possibilidades do uso de cada uma das tecnologias, no fluxo de trabalho empregado no estudo de caso.

\section{REVISÃO TEÓRICA}

Impactos ambientais de obras rodoviárias

O processo de avaliação prévia dos impactos ambientais de um empreendimento, ao utilizar 
informações, estudos, análises e projeções de caráter científico, visa antever com o maior grau de certeza possível os ganhos e perdas ambientais potencialmente decorrentes da implantação de um projeto ou atividade, em curto, médio e longo prazos. A menor interferência ambiental possível em um empreendimento rodoviário é passível de atingimento caso sejam identificados previamente os aspectos ambientais que possam impactar características da área de implantação do empreendimento, devido às atividades de planejamento, instalação e operação (STEINEMANN, 2001).

Impacto ambiental, para Wathern (1988), tem por definição "a mudança em um parâmetro ambiental, num determinado período e numa determinada área, que resulta de uma atividade, comparada com a situação que ocorreria se essa atividade não tivesse sido iniciada". A Resolução CONAMA № 01 de janeiro de 1986, no entanto, define impacto ambiental como:

Qualquer alteração das propriedades físicas, químicas e biológicas do meio ambiente, causada por qualquer forma de matéria ou energia resultante das atividades humanas que, direta ou indiretamente, afetam a saúde, a segurança e o bem-estar da população; as atividades sociais e econômicas; a biota; as condições estéticas e sanitárias do meio ambiente; e a qualidade dos recursos ambientais. (CONAMA, 1986)

Os impactos ambientais de obras rodoviárias necessitam de cuidadosa identificação e categorização dos impactos e se destina a evitar ou mitigar os efeitos de potenciais danos a serem causados no meio ambiente.

\section{Estudo de traçado de obras rodoviárias}

A determinação do traçado é parte essencial do projeto geométrico de uma rodovia. Parâmetros de terreno devem ser considerados, bem como ambientais e sociais, de modo a definir a diretriz de acordo com as limitações impostas pelo Manual de Projeto Geométrico de Rodovias Rurais (BRASIL, 1999).

As alternativas de traçado avaliadas durante o estudo locacional impactam diretamente a qualidade final da via a ser construída, pois a escolha depende do padrão das alternativas disponíveis e, consequentemente, dos parâmetros utilizados para tal escolha. Portanto, é primordial a consideração de aspectos ambientais, econômicos e sociais de maneira conjunta na definição do traçado (STEINEMANN, 2001).

Parte integrante do projeto preliminar de uma rodovia, o Estudo de Traçado tem por objetivo delimitar locais convenientes para passagem da via, usualmente envolvendo etapas de reconhecimento e exploração de modo a resultar em alternativa viável técnica e economicamente. Para a realização de um estudo de traçado são necessários dados precisos dos aspectos geológicos, geotécnicos, hidrográficos, fluviométricos, socioeconômicos e ambientais da área em estudo. Nessa fase é realizada a identificação de alternativas de traçado e possíveis diretrizes, além do estabelecimento de critérios para a via, criação de planos funcionais preliminares e uma avaliação comparativa das opções elencadas (LEE, 2013).

\section{Sistemas de Informação Geográfica (SIG)}

Tecnologia utilizada para auxílio à criação de projetos na área de infraestrutura, especialmente com relação a transportes, são os Sistemas de Informação Geográfica (SIG). Burrough (1986) definiu SIG como um 
conjunto poderoso de ferramentas para coletar, armazenar, recuperar, transformar e visualizar dados sobre o mundo real. Esses sistemas realizam o tratamento computacional de dados geográficos através de sua localização espacial e com base em suas características alfanuméricas, de modo que o administrador tem acesso a todas as informações disponíveis com sua localização na superfície terrestre representada em projeção cartográfica (DAVIS et al., 2001).

SIG é, portanto, um sistema de informações capaz de realizar análises espaciais e operar dados georreferenciados para manipulação, armazenamento e atualização. Um sistema de informações georreferenciadas completo necessita de uma base de dados geográficos com programas capazes de gerenciar os dados físicos, profissionais capacitados para operar esses sistemas, hardware e organização (FERREIRA, 2006).

Os dados geográficos podem ser representados de duas formas: a matricial e a vetorial. Os dados matriciais, também conhecidos como raster, utilizam a organização celular para armazenar os dados, visto que cada organização é 'caracterizada por uma matriz de células de tamanhos regulares, onde cada célula é associada ao conjunto de valores representando as características geográficas da região correspondente' (BOTELHO, 1995).

No que se refere aos dados vetoriais, estes podem ser definidos como elementos de dados que permitem descrever posição e direção através de feições representadas por linha, ponto e polígono (BORGES, 2002). O ponto é um par ordenado $(x, y)$ de coordenadas espaciais, que é utilizado para identificar localizações ou ocorrências no espaço como, por exemplo, a localização de uma cidade. As linhas são definidas por um conjunto de pontos conectados, e são utilizadas para guardar feições unidimensionais, como por exemplo, rodovias e cursos hídricos. Por fim, polígonos são definidos por uma região do plano limitada por uma ou mais linhas poligonais conectadas de tal forma que o último ponto de uma linha seja idêntico ao primeiro da próxima, sendo um exemplo a delimitação da área de uma cidade (FERREIRA, 2006).

As tecnologias BIM e SIG possuem como similaridade o fato de trabalharem com base em banco de dados integrados ao projeto, e embora tenham como foco resultados distintos, a união das duas tecnologias pode trazer facilidades durante o curso de um projeto de infraestrutura (FERREIRA et al., 2012).

\section{METODOLOGIA}

Para elaboração desta pesquisa, executou-se um Estudo de Caso de Avaliação de Impacto Ambiental vinculado a um Estudo Locacional para determinação da alternativa de traçado, com menor impacto ambiental para subtrecho rodoviário.

Primeiramente foram definidos os dados necessários à geração de alternativas de traçado pelos fluxos de trabalho empregados, como o local de implantação do estudo de traçado, as restrições ambientais da área, e realizado um levantamento dos dados matriciais e vetoriais disponíveis.

O subtrecho utilizado como Estudo de Caso nesta dissertação é parte da rodovia federal denominada BR 135/BA entre os km 207 a 212, localizado no estado da Bahia, município de São Desidério. A área é de formação cárstica com alto potencial espeleológico. 
Características técnicas de geometria foram estabelecidas para elaboração do estudo de traçado. 0 trecho foi classificado como uma via arterial secundária de Classe II, pois possibilita o tráfego intermunicipal e serve cidades acima de 10.000 habitantes, alimentando o sistema arterial principal. As larguras adotadas para projeto foram 3,6 metros para pista de rolamento e 2,5 metros para o acostamento.

Os eixos estudados levaram em consideração a adoção de inclinações de talude de 1:1 em relação a cortes e de 1.5:1 para os aterros projetados, devidos às características da geomorfologia local, de relevo montanhoso. A plataforma de pavimentação foi projetada com as larguras e inclinações supracitadas, com adição de 1 metro de cada lado do pavimento prevendo-se a instalação de dispositivos de drenagem superficial.

A Faixa de Domínio considerada engloba, a área das pistas de rolamento, obras de arte, acostamentos, sinalização e faixa lateral de segurança até o alinhamento das cercas que separam a estrada dos imóveis marginais à via ou da faixa de recuo, respeitando minimamente a faixa não edificável de 15 metros de cada lado da rodovia prevista pela Lei Federal de Parcelamento de Solo no 6.766 de 19 de dezembro de 1979. Dessa forma, considerou-se o valor de 60 metros para faixa de domínio devido à extensão dos offsets de terraplenagem, sendo 30 metros para cada lado do eixo. A Tabela 1 apresenta as principais características geométricas adotadas para criação dos traçados.

Tabela 1: Características técnicas do segmento estudado.

\begin{tabular}{l|l}
\hline Velocidade diretriz & $50 \mathrm{~km} / \mathrm{h}$ \\
\hline Raio mínimo de curva horizontal & $80 \mathrm{~m}$ \\
\hline Taxa de superelevação máxima & $8 \%$ \\
\hline Rampa máxima & $7 \%$ \\
\hline Valor K mínimo para curvas convexas & 10 \\
\hline Valor K mínimo para curvas côncavas & 12 \\
\hline Largura da faixa de trânsito & $3,6 \mathrm{~m}$ \\
\hline Largura do acostamento & $2,5 \mathrm{~m}$ \\
\hline
\end{tabular}

A segunda etapa da metodologia se refere ao projeto das alternativas em si, onde são empregadas as tecnologias de modelagem de informação e sistemas de informação geográfica como subsídio à determinação do projeto mais viável para os dados específicos coletados, considerando-se critérios préestabelecidos pelo IBAMA (2018) para o segmento em estudo.

Buscou-se a criação de alternativas de traçado empregando diferentes tecnologias, com objetivo de determinar um fluxo de trabalho que permita a avaliação paramétrica dos dados relativos a cada critério analisado. A fim de facilitar o entendimento das análises realizadas convencionou-se denominar as metodologias empregadas como 'SIG' e 'BIM', de modo a representar a tecnologia aplicada em cada fluxo de trabalho para otimização da análise de impactos na geração do traçado locacional. O fluxograma da Figura 1 apresenta as ferramentas utilizadas na concepção de projeto de cada uma das alternativas.

A Alternativa em BIM buscou a adequação de um corredor tridimensional ótimo, diretamente no software de modelagem de informação. O algoritmo de otimização de corredor age como se aplicasse o método de AIA de 'superposição de cartas' em conjunto com o método de 'redes de interação' por utilização de tecnologia computacional 3D paramétrica e pesos para as áreas de restrição. No software BIM Infraworks 
360 da Autodesk, as camadas de dados foram adicionadas mediante atribuição de pesos e parâmetros máximos, para otimização de traçado, custos e perfil vertical.

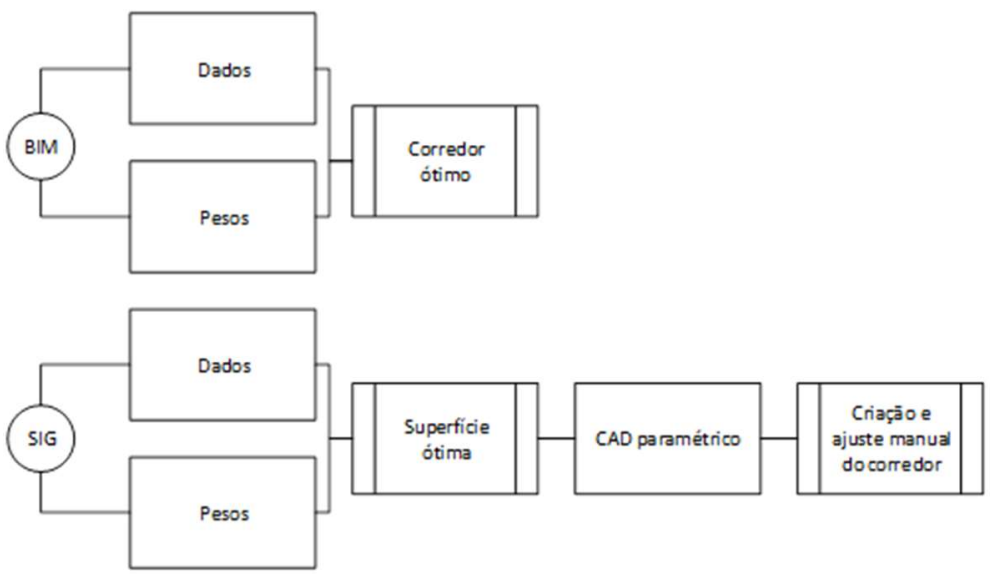

Figura 1: Fluxo de trabalho.

A alternativa em SIG foi determinada pela utilização do software ESRI ArcMAP, com geração de uma superfície otimizada de custos ambientais, a partir da atribuição de pesos para os critérios cujos dados são vetoriais ou matriciais (ferramenta 'cost-benefit analysis'). Uma vez formada a superfície, ela foi importada para um software de projeto geométrico paramétrico (AutoCAD CIVIL 3D). No software paramétrico, considerou-se então o caminho apresentado com melhor custo-benefício ambiental para criação do eixo da alternativa, ou seja, menor interceptação de áreas com critérios restritivos socioambientais.

\section{RESULTADOS}

A fase de estudos ambientais, embora seja anterior ao projeto, necessita de definições geométricas para concepção do projeto, no fluxo de trabalho adotado nessa dissertação. A definição de características técnicas básicas para elaboração do estudo de traçado fez-se importante de modo a permitir que a Avaliação de Impactos Ambientais para que se realizasse concomitantemente o estudo das alternativas locacionais. Desse modo, a seguir descrevem-se as características técnicas utilizadas para projeto geométrico dos corredores gerados.

\section{Restrições socioambientais}

Com relação aos critérios de restrições socioambientais, para a Avaliação de Impactos Ambientais das alternativas, fez-se necessária a consideração de restrições socioambientais, que serviram de base comparativa para definição do traçado de menor impacto. Esta fase remete ao método de avaliação de impacto ambiental de checklist, pois define os itens com possível impacto ambiental.

Por haver critérios previamente determinados pelo órgão ambiental para o segmento em estudo, optou-se por utilizar as restrições socioambientais definidas pelo IBAMA como os critérios de avaliação nos traçados gerados por este Estudo de Caso.

Quanto aos dados geográficos, procedeu-se a obtenção de dados vetoriais, matriciais, estatísticos e sociais sobre os critérios a serem englobados nas análises. A fim de obter os resultados para este estudo de 
caso foram utilizados dados do Banco de Dados Geográficos do Exército Brasileiro (BDGEx, 2019), que é o sistema computacional responsável pelo acesso centralizado às bases de dados geoespaciais distribuídas, produzidas ou adquiridas pela Diretoria de Serviço Gráfico (DSG), responsável pelo mapeamento sistemático brasileiro. A precisão dos dados atende a escala 1:25.000, enquadrada no Padrão de Exatidão Cartográfica (PEC), classe A, seguindo as diretrizes e análises propostas no Decreto no 89.817, de 20 de junho de 1984, que estabelece as instruções reguladoras das normas técnicas da cartografia nacional.

As demais fontes dos dados matriciais e vetoriais empregadas neste estudo foram obtidas através dos seguintes órgãos oficiais competentes: Hidrografia - Agência Nacional das Águas (ANA) -2018; Pedologia nos casos mais abrangentes - Serviço Geológico do Brasil (CPRM) - 2015; Pedologia- RADAMBRASIL - 2003; Unidades de Conservação e uso do solo - World Wide Fund for Nature (WWF Brasil) - 2019; Unidades de Conservação - Instituto Brasileiro do Meio Ambiente e dos Recursos Naturais Renováveis (IBAMA) - 2018; Dados climáticos - Instituto Nacional de Meteorologia (INMET) - 2018; Áreas prioritárias de conservação da biodiversidade - Ministério Meio Ambiente (MMA) - 2018; Registros arqueológicos - Base cartográfica do cadastro nacional de sítios arqueológicos georreferenciados (CNSA) - 2019; Caracterização do patrimônio espeleológico do Instituto Tecnológico de Transportes e Infraestrutura da UFPR (ITTI/UFPR) - 2018.

\section{Pesos para otimização}

Os critérios socioambientais definidos pelo IBAMA como de importante avaliação no segmento tiveram pesos atribuídos para a as análises realizadas. Devido a existência de algoritmos de otimização nos fluxos de trabalho em BIM e SIG, os pesos foram atribuídos previamente à elaboração do traçado, de modo que a otimização levou em conta as áreas de restrição impostas pelos pesos.

A cada camada adicionada aos modelos pode-se atribuir um valor de restrição, que indica ao sistema que evite a alocação do eixo sobre regiões com essa parametrização. Os pesos foram diferenciados para situações também diversas, aquelas que podem ser revertidas ou mitigadas por obras de engenharia, das que não possuem mitigação possível. Foi utilizado ainda como critério a quantidade de terraplenagem necessária, atribuindo-se valores para as diferentes declividades de terreno. Os pesos variam de 0 a 100 , sendo 0 o desejável e 100 totalmente restritivo.

Desse modo, a instabilidade por exemplo, recebeu pesos diferenciados para instabilidade por subsidência, erosão e movimentos de massa, dadas as suas diversas características levando, mais uma vez em consideração as técnicas de mitigação ou minimização dos impactos. A declividade recebeu pesos em função da classe da rodovia a ser traçada, que tem por rampa máxima 7\% de declividade.

Assim, o terreno com inclinação natural de até $7 \%$ deve ser o local preferencial para passagem da via, recebendo peso 0 . As declividades superiores foram divididas em 5 categorias, visando os custos envolvidos de terraplenagem para implantação e áreas de empréstimo ou bota-fora.

A área urbana consolidada e perímetro urbano receberam pesos diferentes pois, embora o perímetro urbano constitua-se em área de expansão da cidade, não há em sua totalidade edificações construídas e infraestrutura implantada, resultando em custos de desapropriação diferenciados para as duas categorias. 
As Áreas de Preservação Permanente (APP) consideradas nos modelos constituem as margens de cursos d'água, como previamente explicitado. Assim, para consideração pelo algoritmo de otimização, o peso utilizado foi 70, de modo a permitir apenas a implantação das cabeceiras de pontes no local, e não que o traçado se desenvolva sobre as APPs.

No que se refere às cavidades, aplicou-se o peso de $70 \mathrm{em}$ um raio de 250 metros de cada cavidade cadastrada no CECAV (em seguimento à normativa CONAMA 347/2004). Utilizou-se de tal parâmetro para que o algoritmo de otimização desvie prontamente da região de mais intensa concentração espeleológica. A Tabela 2 apresenta os valores de parâmetros restritivos utilizados em cada otimização.

Tabela 2: Valores de parâmetros restritivos utilizados nas otimizações.

\begin{tabular}{|c|c|c|c|}
\hline \multicolumn{2}{|c|}{ Parâmetros otimizados } & \multirow{2}{*}{\begin{tabular}{|l|} 
Restrições SIG \\
100 \\
\end{tabular}} & \multirow{2}{*}{\begin{tabular}{|l|} 
Restrições BIM \\
100
\end{tabular}} \\
\hline \multirow{19}{*}{ 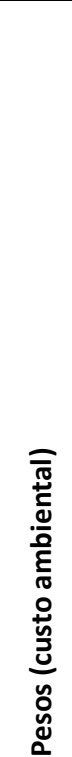 } & Instabilidade por subsidência & & \\
\hline & Área urbana consolidada & 100 & 100 \\
\hline & Patrimônio histórico & 100 & 100 \\
\hline & APA São Desidério & 100 & 100 \\
\hline & Áreas de várzeas e baixadas & 90 & Não aplicável \\
\hline & Instabilidade por movimentos de massa & 90 & 90 \\
\hline & APP & 70 & 70 \\
\hline & Cavidades cadastradas (250 $\mathrm{m}$ do eixo) & 70 & 70 \\
\hline & Perímetro urbano & 70 & 70 \\
\hline & Suscetibilidade a erosão & 60 & 60 \\
\hline & Afloramento rochosos (lapiás) & 50 & 50 \\
\hline & Vegetação nativa & 50 & 50 \\
\hline & Hidrografia & Não aplicável & 50 \\
\hline & Declividade do terreno de 0 a $7 \%$ & 0 & Não aplicável \\
\hline & \begin{tabular}{|l|} 
Declividade do terreno de 7 a $10 \%$ \\
\end{tabular} & 50 & Não aplicável \\
\hline & Declividade do terreno de 10 a $14 \%$ & 60 & Não aplicável \\
\hline & Declividade do terreno de 14 a $20 \%$ & 70 & Não aplicável \\
\hline & \begin{tabular}{|l|} 
Declividade do terreno de 20 a $30 \%$ \\
\end{tabular} & 80 & Não aplicável \\
\hline & Declividade do terreno de acima de $30 \%$ & 90 & Não aplicável \\
\hline \multicolumn{2}{|c|}{ Velocidade diretriz } & Não aplicável & Definida em $80 \mathrm{~km} / \mathrm{h}$ \\
\hline \multicolumn{2}{|c|}{ Nivelamento lateral (limite de eixo) } & Não aplicável & 10 metros \\
\hline \multicolumn{2}{|c|}{ Inclinação de corte } & Não aplicável & $1,0: 1,0$ \\
\hline \multicolumn{2}{|c|}{ Inclinação de aterro } & Não aplicável & $1,5: 1,0$ \\
\hline \multicolumn{2}{|c|}{ Raio mínimo } & Não aplicável & 280 metros \\
\hline \multicolumn{2}{|c|}{ Nivelamento máximo } & Não aplicável & $4,50 \%$ \\
\hline
\end{tabular}

\section{Geração de alternativas}

A etapa seguinte consistiu na elaboração dos traçados por diferentes fluxos de trabalho. A seguir se encontram detalhados os resultados obtidos usando-se cada uma das tecnologias.

\section{Alternativa por Sistema de Informação Geográfica (SIG)}

De acordo com explicado anteriormente, a alternativa com o uso de Sistemas de Informação Geográfica foi criada com a utilização de uma ferramenta de análise de superfícies com pesos e utilizando como base os critérios selecionados na Etapa I, como se aplicando o método de AlA de superposição de cartas.

Na plataforma SIG empregada, ArcMAP, os dados das restrições existentes foram transformados em superfície tridimensional para a determinação do caminho ótimo, que posteriormente teve seu traçado corrigido para a adequação das características técnicas da via. 
A partir da aplicação dos pesos adotados em cada uma das camadas de dados vetoriais ou matriciais para a incorporação no software SIG, e utilizando da ferramenta de análise de custo-benefício ambiental sobre álgebra de mapas, obteve-se a superfície otimizada apresentada na Figura 2. Na figura podem-se observar que as regiões marcadas em tonalidade verde apresentam melhor relação custo benefício em função dos pesos empregados,

Faz-se importante ressalta que não se trata de uma relação monetária quando se utiliza a terminologia 'custo', mas sim de uma terminologia de quantificação do custo ambiental em forma de dano, apresentado pela quantidade (em área) de cada restritivo multiplicado por seu peso.

O inverso é válido para o benefício, pois apresenta menor pontuação na escala, seja por haver menor área interceptada ou menor peso adotado para o critério restritivo. A combinação de todas as camadas versus todos os pesos resulta, portanto, na superfície gerada.

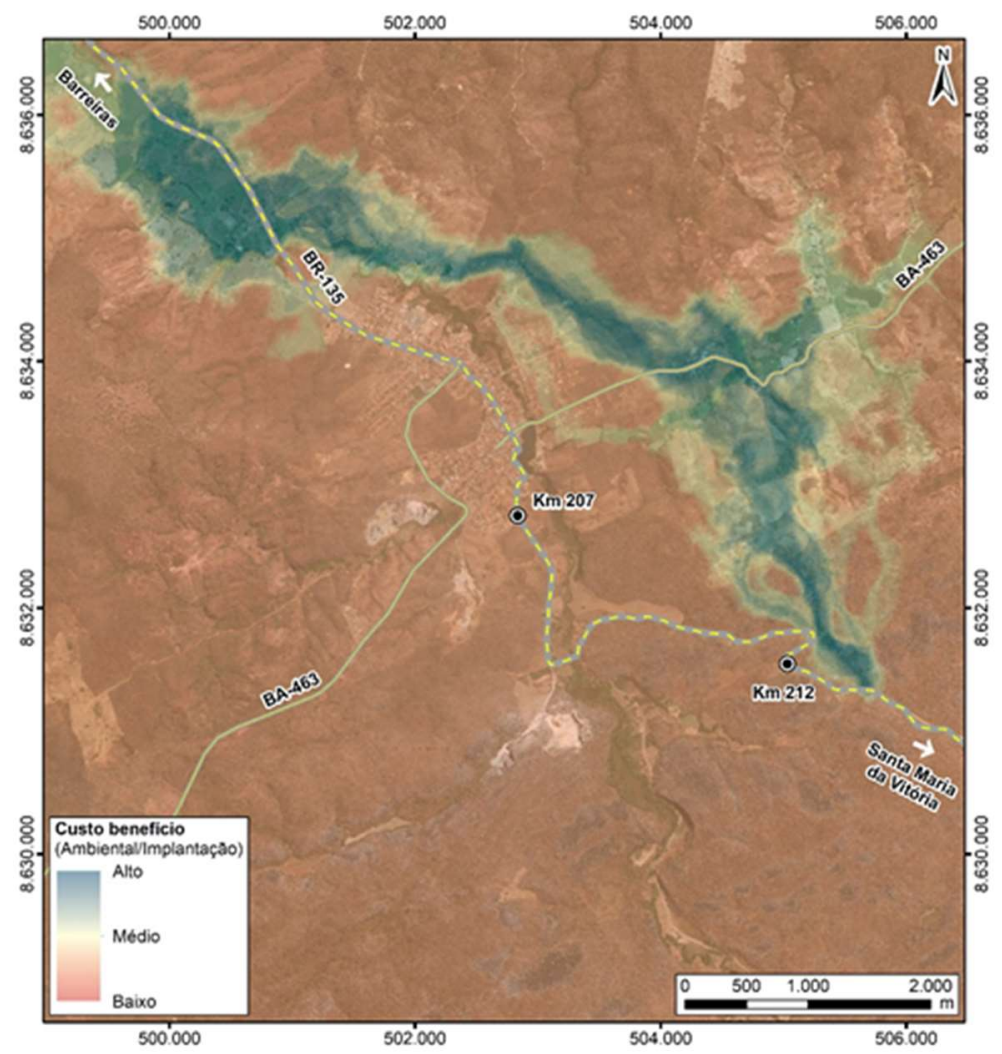

Figura 2: Superfície de custo-benefício ambiental gerada com SIG.

A transposição desta superfície para o software de desenho geométrico paramétrico AutoCAD CIVIL 3D permitiu a criação e um eixo de traçado pelo local de melhor custo-benefício ambiental, observando-se os padrões geométricos da classe da rodovia projetada. Os valores obtidos para a alternativa gerada são apresentados na Tabela 3.

Tabela 3: Quantitativos alternativa SIG.

\begin{tabular}{l|l|l}
\hline Critérios avaliados & Und. & $\boldsymbol{A}$ \\
\hline Transposição de cursos hídricos permanentes & und. & 0 \\
\hline Extensão de transposição de áreas de várzeas & $\mathrm{m}$ & - \\
\hline Extensão estimada de pontes & $\mathrm{m}$ & - \\
\hline Interferência em APP & ha. & 0,45 \\
\hline Área estimada de supressão de vegetação nativa & ha. & 18,69 \\
\hline
\end{tabular}




\begin{tabular}{|c|c|c|}
\hline Interferência em áreas cársticas & ha. & 42,51 \\
\hline Interferência em áreas de instabilidade geológico-geotécnicas & ha. & 0,17 \\
\hline Custo estimado & Milh R\$ & 28,25 \\
\hline Interceptação de áreas urbanas & ha. & 5,57 \\
\hline Quantitativo de realocação de população & hab. & 20 \\
\hline Área total das edificações a serem desapropriadas & $m^{2}$ & 416 \\
\hline Edificações a serem desapropriadas & und. & 5 \\
\hline Interceptação e terras indígenas, quilombolas & ha. & - \\
\hline Interferências com patrimônio artístico e cultural & und. & - \\
\hline Interferências sobre áreas de uso comum utilizadas por comunidades tradicionais e extrativistas & $\mathrm{m}^{2}$ & - \\
\hline Raio mínimo & $\mathrm{m}$ & 140 \\
\hline Rampa máxima & $\%$ & 6 \\
\hline Volume de material movimentado (corte) & $10^{3} \mathrm{~m}^{3}$ & 585,55 \\
\hline Volume de material movimentado (aterro) & $10^{3} \mathrm{~m}^{3}$ & 446,8 \\
\hline Extensão total de aterros & $\mathrm{km}$ & 4 \\
\hline
\end{tabular}

\section{Alternativa por Modelagem de Informações (BIM)}

A Alternativa em tecnologia BIM busca realizar a mesma otimização da criada com SIG, porém diretamente no software de modelagem de informação, como se aplicando o método de AIA de superposição de cartas, em conjunto com o método de redes de interação por utilização de tecnologia computacional 3D paramétrica e algoritmos de otimização.

No software BIM Autodesk Infraworks 360, as camadas de dados foram adicionadas mediante atribuição de valores, pesos e parâmetros máximos, para $\mathrm{A}$ otimização de traçado, custos e perfil vertical.

A partir da utilização dos dados vetoriais e matriciais incorporados diretamente ao software BIM, os pesos foram atribuídos a partir de sua prioridade de conservação, ou seja, áreas mais sensíveis foram consideradas com pesos mais altos. A manutenção de características que não podem ser mitigadas por obras de engenharia e alternativas tecnológicas, ou que resultam em altos custos, foram consideradas com maiores pesos restritivos.

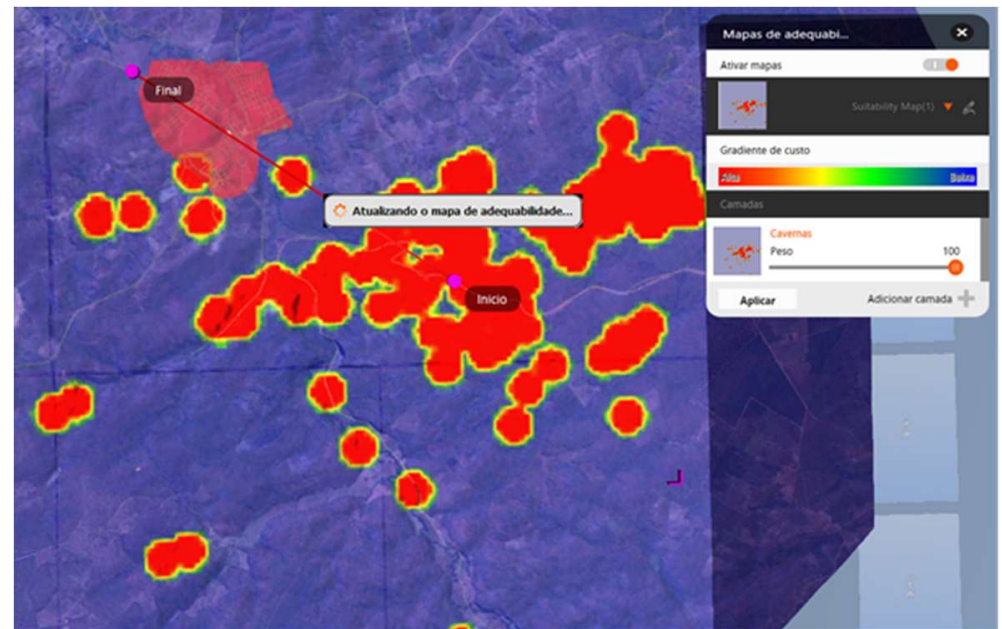

Figura 3: Análise de otimização do traçado não finalizada.

Por se tratar de um software paramétrico com atribuição e informações aos componentes de projeto, a otimização de corredor requer a determinação de parâmetros relativos não somente aos componentes ambientais da região, mas também parâmetros geométricos relativos à classe de projeto da via (Classe II).

Entretanto, a ferramenta de análise do software não suportou a quantidade de camadas necessárias à análise, não gerando um traçado. A Figura 3 representa o status do software (inábil a completar a 
otimização do corredor). Desse modo, não foram possíveis a criação do corredor nem a extração de quantitativos de impactos aos critérios socioambientais considerados.

\section{DISCUSSÃO}

Primeiramente deve ser considerada a ressalva de que, embora as alternativas estudadas tenham sido denominadas 'SIG' e 'BIM', isso se deu unicamente com propósito de facilitar o entendimento das análises realizadas, de modo a representar a tecnologia majoritariamente aplicada em cada fluxo de trabalho para otimização da análise de impactos na geração do traçado locacional. Entretanto, para a geração de cada alternativa várias tecnologias foram utilizadas, sendo todos os dados empregados em SIG. Desse modo, os fluxos de trabalho apresentados foram desenvolvidos utilizando-se da integração de SIG com programas paramétricos de tecnologia BIM, mesmo o caso denominado 'BIM'. Ainda, a alternativa denominada 'SIG', teve a análise de corredor realizada em software de tecnologia SIG, porém foi concluída também com uso de software de desenho gráfico paramétrico, o AutoCAD CIVIL 3D.

Assim, embora os fluxos de trabalho estudados tenham sido diferenciados, de maneira literal podese considerar que as alternativas estudadas se baseiam na aplicação de tecnologias BIM e SIG de maneira conjunta para a avaliação de impactos ambientais de estudo de traçado rodoviário, por meio do estudo de caso de trecho da BR-135/BA.

Necessário, contudo, se faz ressaltar que um dos fluxos empregados neste trabalho, referente a aplicação de um software de modelagem paramétrica 3D de tecnologia BIM, integrado a um sistema SIG para Avaliação de Impacto Ambiental em estudo de traçado rodoviário, não possibilitou a geração de um traçado otimizado pelo software Infraworks, contendo as camadas de restrições necessárias. Devido à limitação da área analisada, bem como quantidade de restritivos ambientais e da capacidade de processamento de dados em nuvem pela ferramenta de otimização paramétrica no software BIM empregado, não foi possível gerar uma alternativa locacional com o programa Infraworks. Assim, a hipótese inicialmente levantada, relativa à possível superioridade qualitativa de análises em BIM não pôde ser comprovada pela utilização do Infraworks. A mesma não pode, todavia, ser também totalmente refutada, visto que os materiais e métodos empregados por esta pesquisa fizeram uso de sistemas BIM advindos de um único fornecedor (Autodesk), para um único estudo de caso.

Pôde-se perceber que a ferramenta de otimização presente no software BIM utilizado permite a consideração de maior variedade de parâmetros do que se pode analisar pelas camadas de dados geoespaciais utilizadas em SIG. Essa capacidade tem sua funcionalidade reduzida, porém, devido à limitação no número de camadas que se analisa simultaneamente.

Enquanto com SIG todas as entradas de dados foram otimizadas em uma única superfície, o software BIM utilizado não pôde processar mais de 4 camadas em uma mesma análise. Este fato implica em impossibilidade de processamento de todas as camadas de dados, uma vez que o algoritmo de otimização do software não permite fazer a análise por partes. Não havendo possibilidade de se processar todas as camadas de dados necessárias, percebe-se que embora haja potencial na ferramenta, para os usos propostos 
neste trabalho a mesma não foi adequada.

Com relação ao fluxo de trabalho empregado e às condições de análise dos impactos gerados pelas alternativas projetadas, notou-se que os critérios de camadas adicionadas às ferramentas de análise não são os mesmos dos aspectos ambientais considerados na AIA tradicional. Portanto, a concepção do projeto de alternativas não gera resultados diretos de impactos, tal como uma avaliação tradicional geraria, mas sim um valor normalizado, relativo aos pesos socioambientais considerados.

Sobre a instituição de pesos de importância relativa aos critérios ambientais considerados na otimização, se faz importante a ressalva de que não há metodologia existente que preconize um padrão para adoção de tais valores. A determinação destes pesos modifica inteiramente a análise, sendo extremamente subjetiva e implicando inclusive em questões éticas, relativas à legitimidade de determinação de importâncias diversas para os atributos analisados.

Este fato, contudo, não desqualifica o trabalho de Avaliação de Alternativas de Traçado de uma rodovia, desde que seja realizado por equipe interdisciplinar e de especialistas. Evitando, desta forma tendências não adequadas.

Com relação aos fluxos de trabalho empregados em relação aos resultados obtidos, percebe-se que a alternativa gerada em SIG possui potencial de englobar mais camadas de análise do que se pode analisar simultaneamente, por um operador humano.

Entretanto, não se pode de maneira alguma concluir que a análise de otimização de traçados de rodovia comparativo por métodos de análise multicritério possa substituir as metodologias tradicionais de criação de traçados e avaliação de impactos ambientais, mas sim que se faz de extrema importância da aplicação de uma otimização para análise prévia, devendo a determinação final do traçado usufruir de ambos os métodos, para se determinar o traçado de menor dano e se obter detalhamento preciso dos impactos gerados, possibilitando a implementação de programas de mitigação.

Desse modo, ressalta-se a eficácia da aplicação da otimização de superfície em plataforma SIG para a geração do traçado, de forma a minimizar os impactos decorrentes de sua implantação.

\section{CONCLUSÕES}

Com relação aos objetivos estabelecidos nesta pesquisa, realizou-se a aplicação de tecnologias BIM e SIG na Avaliação de Impacto Ambiental em estudo de traçado rodoviário, por meio de estudo de caso de subtrecho da BR-135/BA. O fluxo de trabalho para geração de alternativas locacionais em programas paramétricos foi testado resultando em insuficiência da capacidade de processamento do sistema de modelagem BIM empregado, porém gerando resultados com a utilização de sistema SIG.

Verificou-se que a utilização de metodologia com modelagem BIM para estudo de alternativas locacionais considerando-se fatores ambientais necessita ainda de maior desenvolvimento, em se tratando do software utilizado (Autodesk Infraworks 360). Percebe-se que a capacidade processual de SIG é mais ampla, podendo analisar quantidade maior de camadas de dados, porém não suporta componentes paramétricos, sendo necessária a interoperabilidade de dados entre sistemas para finalização da concepção 
do projeto.

Ainda, é importante ressaltar que a criação da superfície de custos ambientais depende inteiramente dos critérios de entrada de dados e dos pesos adotados, de modo que mesmo se utilizando otimizações de dados a avaliação dos impactos não deixa de ser subjetiva, dependendo do crivo do analista responsável. Embora as tecnologias apresentem grande potencial analítico, as mesmas servem exclusivamente ao propósito de ferramentas, sendo necessário conhecimento técnico do operador para que a análise gerada seja coerente com o esperado.

Ressalta-se que para o segmento utilizado no estudo de caso já haviam sido definidos pelo IBAMA as variáveis ambientais a serem analisadas na fase de estudo de traçado rodoviário. Mas, a avaliação de quais variáveis devem ser consideradas na otimização, bem como quais os pesos a serem adotados, sendo parte essencial da otimização de traçado.

Outra consideração importante refere-se à qualidade dos dados inseridos no programa de otimização. Sendo uma fase anterior à aprovação do projeto, a fase de estudo locacional trabalha predominantemente com dados secundários. Assim, a procedência, escala e precisão dos dados disponíveis afeta consideravelmente a qualidade do resultado obtido. Para o estudo de caso realizado, foram encontradas diversas fontes de dados oficiais da região, porém em regiões menos documentadas o método pode se mostrar inviável sem que sejam coletados dados primários.

Conforme apresentado no tópico de discussões, observa-se que outra conclusão importante deste trabalho se refere à necessidade de aplicação de mais um método de avaliação de impacto ambiental na determinação do traçado rodoviário, visto que a aplicação conjunta de metodologias e tecnologias com diferentes focos aumenta a densidade da análise e melhora seus resultados. A aplicação de otimização de dados com análise multicritério resulta na determinação do traçado de menor impacto enquanto a aplicação de método tradicional de AIA como matriz de avaliação de impactos permite a determinação dos impactos a serem monitorados e mitigados, englobando as ações necessárias à redução dos danos causados pelo empreendimento.

Com relação às tecnologias testadas, percebe-se que mesmo sem a utilização de modelagem paramétrica em BIM, a qual não apresentou capacidade de processamento suficiente, a otimização de superfície em plataformas SIG permite entradas de dados com ajuste de pesos, tecnologia que se mostrou mais apta à otimização.

Devido à subjetividade da escolha dos pesos aplicados às variáveis ambientais, seria de grande utilidade a existência de um método tal qual a matriz de AIA para determinação dos pesos e geração de alternativas otimizadas. Logo, se faria possível a compatibilização dos aspectos geradores de impactos com os critérios de entrada de dados nos sistemas de elaboração de projeto, a fim de adiantar a AIA para a etapa de concepção.

Desse modo, como recomendação para estudos futuros, a fim de viabilizar a utilização de modelagem de otimização, se faz importante a criação de uma metodologia para determinação dos pesos a serem empregados às variáveis ambientais na análise multicritério que inclua em suas análises a questão da 
legitimidade da atribuição de importâncias com pesos relativos para camadas de dados que representem elementos dos meios físico, biótico e socioeconômico.

Estes, no entanto, adequados a cada projeto rodoviário, atendendo suas peculiaridades e necessidades. Usualmente, cada trecho é um e único desafio. Também como recomendação para estudos futuros é interessante ressaltar que este estudo aplicou ferramentas BIM de um único desenvolvedor (Autodesk), sendo interessante a comparação com o potencial de análise de programas de otimização BIM de outros desenvolvedores.

Como conclusão final, percebe-se por meio deste trabalho a existência de potencial para o desenvolvimento de metodologias de implementação de tecnologias de dados georreferenciados e otimização de traçados rodoviários já existentes no mercado, com a finalidade de avaliação de impacto ambiental. Porém, em função das limitações de processamento atualmente disponíveis e, sobretudo, em função da grande subjetividade envolvida no processo de avaliação de impactos ambientais, se mantém essencial o conhecimento da área em estudo, bem como o crivo e avaliação do analista e utilizador do programa computacional de auxílio à análise.

\section{REFERÊNCIAS}

ANA. Agência Nacional de Águas. Ministério do Meio Ambiente. Conjuntura dos Recursos Hídricos no Brasil. Brasília: ANA, 2018.

BDGEx. Base cartográfica do banco de dados geográficos do exército brasileiro. Brasília: BDGEx, 2018.

BORGES, K. A. V.. Modelagem de Dados Geográficos. Belo Horizonte: UFMG, 2002.

BOTELHO, M. A.. Incorporação de facilidades espaçotemporais em banco de dados orientados a objetos. Campinas: UNICAMP, 1995

BRASIL. Departamento Nacional de Infraestrutura de Transportes. Manual de Projeto Geométrico de Rodovias Rurais. Rio de Janeiro: DNIT, 1999.

BRASIL. Ministério do Meio Ambiente. Base cartográfica de áreas prioritárias para a conservação biodiversidade, cadastro ambiental rural e unidades de conservação. Brasília: MMA, 2018.

CONAMA. Conselho Nacional do Meio Ambiente. Resolução n. 001, de 23 de janeiro de 1986. Dispõe sobre critérios básicos e diretrizes gerais para a avaliação de impacto ambiental. Brasília: CONAMA, 1986.

CONAMA. Conselho Nacional do Meio Ambiente. Resolução CONAMA n. 347, de 10 de setembro de 2004. Dispõe sobre o patrimônio espeleológico. Brasília: CONAMA, 2004.

CNSA. Base cartográfica do cadastro nacional de sítios arqueológicos georreferenciados. Brasília: CNSA, 2019.

CPRM. Base cartográfica de unidades litoestratigráficas. Brasília: CPRM, 2015.
DAVIS, C.; NETO, G. C.. Arquitetura de Sistemas de Informação Geográfica. São José dos Campos: Instituto Nacional de Pesquisas Espaciais, Ministério da Ciência e Tecnologia, 2001.

FERREIRA, B.. Revisão de literatura e análise em planejamento de transportes usando os sistemas de informações geográficas. Monografia (Bacharelado em Engenharia Civil) - Universidade Federal de Viçosa, Viçosa, 2006.

FERREIRA, R. M. P.; FARIA, S. D.. Aplicação de Sistemas de Informações Geográficas na Gestão da Informação e no Planejamento de Sistema Rodoviário. In: SIMPÓSIO BRASILEIRO DE CIÊNCIAS GEODÉSICAS E TECNOLOGIAS DA GEOINFORMAÇÃO, 5. Anais. Recife, 2012.

IBAMA. Instituto Brasileiro do Meio Ambiente e dos Recursos Naturais Renováveis. Termo de Referência SEI/IBAMA 352218. Brasília: IBAMA, 2018.

ITTI/UFPR. Instituto Tecnológico de Transportes e Infraestrutura da Universidade Federal do Paraná. Prospecção e Caracterização do Patrimônio Espeleológico da Área de Influência Direta da Implantação da BR-135/BA: São Desidério/BA (Km 207,0) a Correntina/BA (Km 344,7). Curitiba, 2018.

LEE, S. H.. Introdução ao Projeto Geométrico de Rodovias. Florianópolis: UFSC, 2013.

PIMENTA, A. F. F.; RATTON, E.; BLASI, G. F.; SOBANSKI, M. B.; ALBACH, D. M.. Gestão para o licenciamento ambiental de obras rodoviárias. Curitiba: Departamento de Transportes da Unidade Federal do Paraná, 2014

STEINEMANN, A.. Improving alternatives for environmental impact assessment. Environmental Impact Assessment 
Review, City Planning Program, Georgia Institute of Technology, 2001.
WATHERN, P.. Introductory guide to EIA. Environmental Impact Assessment: Theory and Practice Unwin Hyman. 1988.

A CBPC - Companhia Brasileira de Produção Científica (CNPJ: 11.221.422/0001-03) detém os direitos materiais desta publicação. Os direitos referem-se à publicação do trabalho em qualquer parte do mundo, incluindo os direitos às renovações, expansões e disseminações da contribuição, bem como outros direitos subsidiários. Todos os trabalhos publicados eletronicamente poderão posteriormente ser publicados em coletâneas impressas sob coordenação da Sustenere Publishing, da Companhia Brasileira de Produção Científica e seus parceiros autorizados. Os (as) autores (as) preservam os direitos autorais, mas não têm permissão para a publicação da contribuição em outro meio, impresso ou digital, em português ou em tradução. 